\title{
Echocardiographic and Exercise Test Findings in Patients With Dyslipidemia and Coronary Artery Disease Before and After Treatment With Simvastatin With or Without Fenofibrate
}

\author{
Reza Karbasi-Afshar ${ }^{1}$, Ayat Shahmari ${ }^{2}$, Amin Saburi ${ }^{3,4^{*}}$ \\ ${ }^{1}$ Faculty of Medicine, Baqiyatallah University of Medical Sciences, Tehran, IR Iran \\ ${ }^{2}$ Cardiology Ward, Baqiyatallah Hospital, Tehran, IR Iran \\ ${ }^{3}$ Chemical Injury Research Center, Baqiyatallah University of Medical Sciences, Tehran, IR Iran \\ ${ }^{4}$ Health Research Center, Baqiyatallah University of Medical Sciences, Tehran, IR Iran
}

\section{A R T I C L E I N F O}

Article type:

Original Article

Article history:

Received:1 Jan 2011

Revised: 1 Jul 2011

Accepted:17 Jul 2011

\section{Keywords:}

Coronary Artery Disease

Myocardium

Dyslipidemias

Simvastatin

Fenofibrate

\begin{abstract}
A B S T R A C T
Background: Various treatment protocols for dyslipidemia and coronary artery disease have been suggested. In spite of lipid-lowering effects, various effects of statins and fibrates have been reported in the literature.

objectives: The aim of this study was to assess the cardiac efficacy of Simvastatin with or without fenofibrate on cardiac function.

Patients and Methods: A cohort study was conducted on 124 patients with dyslipidemia and coronary artery disease. Patients were randomly divided into two groups: the first group $(n=64)$ received Simvastatin (60 - $20 \mathrm{mg} /$ day) and fenofibrate (200 $\mathrm{mg} /$ day), and the second group $(n=60)$ received Simvastatin (20 - $60 \mathrm{mg} /$ day) alone. Treatment lasted 1 year, and the patients were evaluated after treatment.

Results: The mean age was $54.3 \pm 6.5$ years, and $53.2 \%$ of patients were male. Compared to baseline, after 12 months of treatment the lipid profiles of both groups decreased significantly $(P<0.05)$. The change in left-ventricular ejection fraction in the first group was statistically significant $(P=0.01)$. The exercise test time and metabolic equivalent of tasks index significantly increased in the first group $(P=0.014, P=0.006)$, but these changes were not significant in the second group $(P=0.289, P=0.744)$.

Conclusions: Lipid-regulating therapies including Simvastatin and fenofibrate improved myocardial function and reduced myocardial ischemia, so combined therapy is recommended for treating dyslipidemia in high-risk patients for cardiovascular problems.

Copyright $\odot 2011$ Kowsar Corp. All rights reserved.
\end{abstract}

Implication for health policy/practice/research/medical education:

This is a challenging issue about the effects of lipid-profile-lowering agents (i.e. fibrates) on myocardial structure and function in patients with coronary artery diseases and dyslipidemia although there are many studies which confirmed the efficacy of these medications on lipid profile via laboratory findings. In this study we focused on the cardiac effect of lipid profile lowering agents via echocardiography and exercise test.

- Please cite this paper as:

Karbasi-Afshar R, Shahmari A, Saburi A. Echocardiographic and Exercise Test Findings in Patients With Dyslipidemia and Coronary Artery Disease Before and After Treatment With Simvastatin With or Without Fenofibrate. Int Cardiovasc Res J.2011;5(4):143-7. DOI:10.5812/icrj.4378

* Corresponding author: Amin Saburi, Chemical Injury Research Center and Health Research Center, Baqiyatallah University of Medical Sciences, Mollasadra St, Vanak Sq, Tehran, IR Iran. Tel: +98-2188600062, +989127376851, Fax:+98-2188600062,E-mail: aminsaburi@yahoo.com.

DOI: 10.5812 /icrj.4378

Copyright @2011 Kowsar Corp. All rights reserved.

\section{Background}

Cardiac events due to coronary artery disease (CAD) are the major cause of death in many countries, particularly among developed countries. In addition, myocardial ischemia and related cardiac complications are among the common insufficiencies of CAD (1) Recent studies have revealed the notable role of hyperlipidemia as a 
prognostic factor in the structure and function of the left ventricle secondary to the myocardial infarction in patients with CAD (2-4) Also, lipid-regulating drugs, particularly those that reduce cholesterol, such as statins, would improve prognosis in patients diagnosed with cardiac ischemia (acute and chronic), and moreover, could be used to treat hypercholesterolemia effectively and safely (5). These studies have confirmed that lipidlowering agents could ameliorate the clinical status and prognosis of CAD patients. Some studies have approved the efficacy of the lipid-lowering agents to decline the frequency of (lethal and nonlethal) acute coronary syndromes (6).

The effects of other lipid-lowering agents, especially those that are effective on triglycerides, are not thoroughly examined for cardiac function and structure in patients diagnosed with cardiac ischemia. Currently, there is much interest on the therapeutic effects of statins along with other triglyceride-lowering agents on myocardial structure and function. There is some evidence on the preventive effects of statins on the myocardial remodeling in cases with recent myocardial infarction or patients with nonischemic cardiomyopathy (7). However, the effects of statins accompanied by other lipid-profile-lowering medicines (i.e. fibrates) on myocardial structure and function in patients with CAD and dyslipidemia have not been clearly established.

\section{Objectives}

The objective of this study was to evaluate the plausible effects of Simvastatin with or without fenofibrate on myocardial structure and function as well as paraclinical findings in patients with CAD and dyslipidemia.

\section{Patients and Methods}

A prospective cohort study was conducted on patients with CAD and dyslipidemia. After 6 weeks of antihyperlipidemia therapy based on findings from the National Cholesterol Education Program of the American Heart Association, the patients were examined again (8). The inclusion criteria were as follows: patients diagnosed with constant CAD with positive exercise test time, total cholesterol $\geq 200 \mathrm{mg} / \mathrm{dL}$, Triglyceride (TG) $\geq 250 \mathrm{mg} / \mathrm{dL}$, low density lipoprotein (LDL) $\geq 100 \mathrm{mg} / \mathrm{dL}$, and normal liver function test. Also, patients diagnosed with myocardial infarction in the last 6 months, chronic heart failure (New York Heart Association Function Class II and higher), heart-valve disease, systolic blood pressure $>170$ $\mathrm{mmHg}$, diastolic blood pressure $>100 \mathrm{mmHg}$, dysrhythmia (atrial flutter, atrio-ventricular block II, and more), uncontrolled insulin-dependent diabetes, and serious liver, kidney, and neoplastic diseases were excluded from the study.

One hundred forty-nine patients meeting the inclusion criteria were randomly divided into 2 groups: the first group received Simvastatin $(20 \mathrm{mg} /$ day $)$ and fenofibrate (200 mg/day) with lipid-restricted diet and exercise, and the second group received Simvastatin (20 mg/day) with the same diet and exercise. The treatment was given for 12 months. Twenty-five patients failed during the follow up. The starting dose of Simvastatin was $20 \mathrm{mg} /$ day. The main objective of this study was to help patients improve their blood-lipid profiles. So, if an LDL level of under 100 was not achieved during the first week, the dose of Simvastatin was increased to $40 \mathrm{mg} /$ day, and if the patient did not respond to this dosage, it was increased to 60 $\mathrm{mg}$ /day after 6 weeks (based on recommendations from the National Cholesterol Education Program (9). A lipid profile and a liver-function test were taken at 1.5, 3, and 12 months.

During the study, all patients received a standard therapeutic regiment consisting of $80 \mathrm{mg} /$ day of aspirin, beta blocker, nitrate, and calcium-channel blocker daily. The therapeutic cardiovascular regiment was not adjusted during the study. Before and after the study (12 months), the same specialist took a lipid profile (LDL, TG, high-density lipoprotein [HDL], and cholesterol), liver-function test, electrocardiography, exercise test time, and Doppler echocardiography from the patients. The study was approved by Baqiyatallah University's Scientific and Ethical committee. The study was funded by the Scientific Research committee of the Medicine Department, and no extra expenses were imposed on the patients. Study participation was voluntary, and patients were free to quit the study at any time. After collecting the data, all the statistical analyses were carried out using SPSS version 17 via paired and independent $t$ tests, and ANOVA and $P$ values of $<0.05$ were considered statistically significant.

\section{Results}

Overall, 124 patients completed the study (age range $=42-56$; mean and standard deviation $=54.3 \pm 5.6$ ). The mean age in the first group (treated with a combination of Simvastatin and fenofibrate) was $52.8 \pm 4.7$ and 53.1\% were male; the mean age in the second group (Simvastatin without fenofibrate) was $53.1 \pm 7.3$ and $53.3 \%$ were male. No significant statistical differences were found for the demographic variables between both groups (Table 1).

The lipid profiles from both groups before and after treatment are listed in Table 2. After 6 weeks of treatment, a desirable lipid profile was achieved in only 25 cases (20.16\%) in both groups, which in these cases Simvastatin was continued $20 \mathrm{mg} /$ day; while in other cases the dose of Simvastatin was increased to $40 \mathrm{mg} / \mathrm{kg}$. After 6 weeks, LDL did not decrease to $100 \mathrm{mg} / \mathrm{dL}$ in 19 cases (15.32\%). At the end of 12 weeks treatment, $4.5 \%$ of all the patients had an LDL measurement of $>100 \mathrm{mg} / \mathrm{dL}$; for these cases, the Simvastatin dose was increased to $60 \mathrm{mg} /$ day and continued up to 12 months. The 12-month therapeutic protocol in the first and second group decreased their total cholesterol by $25.3 \%$ and $12.93 \%$, LDL by $38.97 \%$ and $33.91 \%$, HDL by $23.41 \%$ and $3.80 \%$, and triglycerides by $44.93 \%$ and $3.09 \%$, respectively. Also, the changes from baseline in total cholesterol and LDL in the first group were statisti- 


\begin{tabular}{|c|c|c|c|c|c|c|c|}
\hline \multirow{2}{*}{ Items } & \multicolumn{3}{|c|}{ First Group $(n=64)$} & \multicolumn{3}{|c|}{ Second Group $(n=60)$} & \multirow{2}{*}{$\begin{array}{l}\text { PValue Between } \\
\text { Groups }\end{array}$} \\
\hline & Base Line & 12th mo & PValue & Base Line & 12th mo & PValue & \\
\hline Mean age \pm SD & $52.8 \pm 4.7$ & - & - & $53.1 \pm 7.3$ & - & - & 0.544 \\
\hline Male & 34 & - & - & 32 & - & - & 0.659 \\
\hline Female & 30 & - & - & 28 & - & - & 0.741 \\
\hline Systolic blood pressure, $\mathrm{mmHg}$ & $134.1 \pm 10.1$ & $133.8 \pm 9.3$ & 0.154 & $131.2 \pm 10$ & $131.9 \pm 8.5$ & 0.325 & 0.201 \\
\hline Diastolic blood pressure, $\mathrm{mmHg}$ & $84.4 \pm 4.3$ & $84.3 \pm 3.1$ & 0.847 & $83.9 \pm 4.2$ & $84.2 \pm 5.1$ & 0.265 & 0.754 \\
\hline Smoker & $18.1 \%$ & $18.1 \%$ & - & $17.9 \%$ & $17.9 \%$ & - & 0.841 \\
\hline BMI, Kg/M2 & $28.1 \pm 2.8$ & $28.6 \pm 3.4$ & 0.369 & $27.4 \pm 2.6$ & $27.9 \pm 4.1$ & 0.657 & 0.632 \\
\hline Diabetes mellitus & $13.6 \%$ & $13.6 \%$ & - & $12.8 \%$ & $12.8 \%$ & - & 0.489 \\
\hline Previous MI & $18.2 \%$ & $18.2 \%$ & - & $10.3 \%$ & $10.3 \%$ & - & 0.063 \\
\hline Beta blocker user & $52.3 \%$ & $52.3 \%$ & - & $53.8 \%$ & $53.8 \%$ & - & 0.497 \\
\hline Calcium channel blocker user & $29.5 \%$ & $29.5 \%$ & - & $30.8 \%$ & $30.8 \%$ & - & 0.369 \\
\hline Nitrates user & $15.9 \%$ & $15.9 \%$ & - & $15.4 \%$ & $15.4 \%$ & - & 0.581 \\
\hline ACE inhibitor user & $31.8 \%$ & $31.8 \%$ & - & $30.8 \%$ & $30.8 \%$ & - & 0.807 \\
\hline
\end{tabular}

\begin{tabular}{|c|c|c|c|c|c|c|}
\hline \multirow[t]{2}{*}{ Items } & \multicolumn{3}{|c|}{ First Group } & \multicolumn{3}{|c|}{ Second Group } \\
\hline & Baseline & $12^{\text {th }}$ mo & $P$ Value & Baseline & $12^{\text {th }} \mathrm{mo}$ & PValue \\
\hline Total cholesterol & $230.1 \pm 41.3$ & $172.5 \pm 47.9^{b}$ & $<0.001$ & $231.9 \pm 35.5$ & $201.9 \pm 42.5$ & 0.061 \\
\hline Total triglyceride & $295.1 \pm 41.3$ & $162.5 \pm 47.9^{c}$ & 0.003 & $290.9 \pm 35.5$ & $281.9 \pm 40.5$ & 0.083 \\
\hline $\mathrm{HDL}^{\mathrm{a}}$ & $38.9 \pm 7.3$ & $48.01 \pm 8.1^{c}$ & 0.021 & $39.4 \pm 8.1$ & $40.9 \pm 9.3$ & 0.542 \\
\hline $\mathrm{LDL}^{\mathrm{a}}$ & $147.8 \pm 33.6$ & $90.2 \pm 36.3^{b}$ & $<0.001$ & $150.1 \pm 27.8$ & $99.2 \pm 33.6$ & 0.024 \\
\hline $\mathrm{AST}^{\mathrm{a}}$ & $24.8 \pm 5.3$ & $26.2 \pm 4.6 \mathrm{c}$ & 0.014 & $23.5 \pm 5.2$ & $24.1 \pm 4.1$ & 0.601 \\
\hline $\operatorname{ALT}^{\mathrm{a}}$ & $26 \pm 4.61$ & $26.9 \pm 3.1$ & 0.259 & $25.7 \pm 3.2$ & $26.2 \pm 4.1$ & 0.365 \\
\hline
\end{tabular}

a Abbreviations: ALT, alanine transaminase; AST, aspartate transaminase; HDL,High-density lipoprotein; LDL, Low-density lipoprotein

${ }^{\mathrm{b}} \mathrm{P} 0<0.001$

${ }^{\mathrm{c}} P 0<0.01$

\begin{tabular}{lllll}
\hline \multicolumn{2}{l}{ Table 3. Echocardiographic Findings } & \multicolumn{2}{c}{ First Group } & \multicolumn{2}{c}{ Second Group } \\
\cline { 2 - 3 } \multicolumn{1}{l}{ Items } & \multicolumn{1}{c}{ Baseline } & $\mathbf{1 2}^{\text {th }}$ mo & & $\mathbf{1 2}^{\text {th }}$ month \\
\hline $\mathrm{LV}^{\mathrm{a}}$ ejection fraction, \% & $59.8 \pm 8.04$ & $62.9 \pm 4.43^{\mathrm{b}}$ & $61.1 \pm 3.4$ & $60.04 \pm 6.4$ \\
$\mathrm{LV}$ mass, gr/m & $77.5 \pm 9.7$ & $74.6 \pm 10.4$ & $77.4 \pm 12.2$ & $80.6 \pm 13.6^{\mathrm{c}}$ \\
$\mathrm{E}^{\mathrm{b}} / \mathrm{A}^{\mathrm{c}}$ ratio & $0.97 \pm 0.2$ & $1.01 \pm 0.21$ & $0.98 \pm 0.19$ & $0.97 \pm 0.23$ \\
Isovolumic relation Time, $\mathrm{s}$ & $0.088 \pm 0.02$ & $0.089 \pm 0.01$ & $0.090 \pm 0.02$ & $0.092 \pm 0.01$ \\
\hline
\end{tabular}

a Abbreviation: LV, left ventricular

${ }^{b}$ E, early diastolic transmitral velocity

${ }_{\mathrm{d}}^{\mathrm{c}} \mathrm{A}$, late diastolic transmitral velocity

$\mathrm{d}_{P<0.001}$

$\mathrm{e}_{P}<0.01$

cally significant at $P=0.01$, whereas the triglyceride and HDL changes were significant at $P=0.001$. Nonetheless, no significant changes were detected in the lipid profile of the second group except for LDL amount $(P=.024)$.

At the end of the 12-month study period, diastolic-function parameters (early and late diastolic transmitral velocity, isovolumetric relaxation time) had not changed significantly from baseline in either group Table 3, although the change in left ventricular (LV) ejection fraction in the first group was statistically significant $(P$ $=0.01)$. The LV mass index statistically increased in the second group after the 12-month treatment $(P=0.039)$, whereas no statistically significant changes appeared in the first group. The anti-ischemic effect was investigated with exercise test time, which significantly increased in the Simvastatin-fenofibrate group Table 4. The metabolic equivalent of tasks index increased (4.32 $0.5 \pm$ to $5.68 \pm$ 0.5 ) in the first group, but the changes were not statistically significant in the second group.

\section{Discussion}

The results showed that the therapeutic effect of a Simvastatin-fenofibrate regiment on the lipid profiles and the ejection fraction of the LV increased, whereas patients who received Simvastatin alone showed an increase in the LV size and mass. Additionally, we conclude that a compound regimen containing Simvastatin and 


\begin{tabular}{|c|c|c|c|c|c|c|}
\hline \multirow[t]{2}{*}{ Items } & \multicolumn{3}{|c|}{ First Group } & \multicolumn{3}{|c|}{ Second Group } \\
\hline & Baseline & $12^{\text {th }}$ mo & Pvalue & Baseline & $12^{\text {th }}$ mo & Pvalue \\
\hline Exercise test time (Min) & $5.21 \pm 1.81$ & $6.01 \pm 1.08^{b}$ & $<0.001$ & $5.43 \pm 1.26$ & $5.32 \pm 1.20$ & 0.089 \\
\hline METS $^{a}$ & $4.32 \pm 0.50$ & $5.68 \pm 0.54$ & $<0.001$ & $4.43 \pm 0.92$ & $4.41 \pm 1.11$ & 0.121 \\
\hline
\end{tabular}

a Abbreviation: METS; metabolic equivalents tasks

${ }^{b}{ }_{P}<0.01$

fenofibrate could improve cardiac function compared to a regimen consisting of Simvastatin alone. Moreover, regarding the changes in exercise test time, myocardial ischemia could be lessened more by administering Simvastatin and fenofibrate together than by administering Simvastatin alone. Furthermore, echocardiography findings showed an increase in end-diastolic and systolic volumes and LV mass due to Simvastatin without fenofibrate prescription in comparison with Simvastatin and fenofibrate, which can be responsible for remolding secondary to chronic cardiac ischemia; this finding is compatible with the findings for exercise test time in a study by Mohlenkamp et al. (10).

Some studies have investigated the impacts of these medications on the lipid profiles and heart functioning. Zeman et al. conducted a clinical trial study on 86 patients who were divided into two groups similar to the present study. They demonstrated that long-term treatment (at least 1 year) by one fibrate and statin could conspicuously rectify lipid profiles without any significant changes in hepatic or muscular enzymes. However, they used Pravastatin and ciprofibrate instead of Simvastatin and fenofibrate, and they did not investigate cardiacfunction parameters. Therefore, their findings are not comparable with the findings of the present study (11).

Also, Klosiewicz-Latoszek et al. demonstrated that the combination therapy with Simvastatin and fenofibrate would effectively regulate serum-lipid profiles in patients diagnosed with mixed hyperlipidemia rather than a separate prescription of each, and although cardiac outcomes were not investigated, the results correspond to our study's results (12). Other studies have also emphasized and corroborated the effectiveness of combination of one statin and one fibrate (particularly fenofibrate) to regulate hypercholesterolemia and hypertriglyceridemia, although the impact of this protocol on cardiac function as well as its efficacy on lipid profiles has not been cleared $(13,14)$. In compared to statins, fibrates may increase HDL levels despite a decline in triglycerides. Also, fibrates can increase HDL levels by activating plasmatic lipase lipoproteins (15). Some experimental studies have demonstrated a preventive effect of statins and fibrates on myocardial hypertrophy via reducing atherogenic lipoproteins and inhibiting the synthesis of isoprenoids $(16,17)$. In addition, 12-month monotherapy with Atorvastatin (20-60 mg/day) had no prominent effect on the decline of the LV mass (18).

Myocardial ischemia during daily activities can induce by dysregulation of the vasomotor activity of the coronary artery. Also, an increase in LDL and TG levels may disrupt endothelial activity and lead to coronary artery spasms (19). Therefore, declines in LDL and TG levels and increases in HDL could regulate endothelial activity and prevent coronary disease (20). The effects of statinfibrate protocol on coronary artery blood supply have been affirmed (21). In this study, a significant decrease over 12 months in myocardial ischemia in patients with CAD who underwent fibrate-statin treatment could be a result of establishing endothelium (22).

It seems that some factors are related to the diminution of myocardial ischemia after receiving statin and fibrate more than changes in blood lipids. Recent studies have demonstrated that statins could alleviate endothelial inflammation, atherosclerotic plaque formation, arrhythmias, and increase revascularization (23). Myocardial ischemic is an inflammatory process, and, therefore, the anti-inflammatory effect of statins can be useful for performance and survival of ischemic tissue. However, the effect of fibrates has not yet been approved for controlling inflammations $(24,25)$. It seems that the 1-year therapy by statin plus fenofibrate could have a positive effect on myocardial structure and function, both in cases with CAD and dyslipidemia and in a thorough improvement on blood-lipid profiles. The limitations of the present study were a lack of angiographic findings and supplemental analysis changes to confirm the anti-ischemic effect of this protocol in these patients. We recommend that future studies investigate the efficacy of this therapeutic protocol on cardiac function and atherosclerotic plaque by using angiography in patients diagnosed with combined dyslipidemia.

\section{Acknowledgments}

The authors would like to acknowledge the personnel and colleagues in the cardiovascular clinic of Baqiyatallah for their kind cooperation throughout all stages of the project. We also thank Dr. Ali Haji Hashemi and Dr. Hamed Moghadam Manesh in particular, who has helped us to accomplish the study at hand.

\section{Financial Disclosure}

None declared.

\section{Funding/Support}

None declared.

\section{References}

1. Nakamura M, Yamashita T, Yajima J, Oikawa Y, Ogasawara K, Sagara $\mathrm{K}$, et al. Impact of early statin initiation on secondary preven- 
tion in Japanese patients with coronary artery disease. J Cardiol. 2011;57(2):172-80.

2. Campbell DJ, Somaratne JB, Jenkins AJ, Prior DL, Yii M, Kenny JF, et al. Impact of type 2 diabetes and the metabolic syndrome on myocardial structure and microvasculature of men with coronary artery disease. Cardiovasc Diabetol. 2011;10:80.

3. Moyers B, Farzaneh-Far R, Harris WS, Garg S, Na B, Whooley MA. Relation of whole blood n-3 fatty acid levels to exercise parameters in patients with stable coronary artery disease (from the heart and soul study). Am J Cardiol. 2011;107(8):1149-54.

4. Kerimkulova A, Noruzbaeva A, Lunegova O, Gotfrid I, Mirrakhimov A, Davydova N, et al. Influence of one-year treatment with lovastatin on myocardial remodeling and ischemia in patients with coronary artery disease. Anadolu Kardiyol Derg. 2011;11(1):16-21.

5. Kadota S, Matsuda M, Izuhara M, Baba O, Moriwaki S, Shioji K, et al. Long-term effects of early statin therapy for patients with acute myocardial infarction treated with stent implantation. J Cardiol. 2008;51(3):171-8.

6. Cheung BM, Lauder IJ, Lau CP, Kumana CR. Meta-analysis of large randomized controlled trials to evaluate the impact of statins on cardiovascular outcomes. Br J Clin Pharmacol. 2004;57(5):640-51.

7. Liu M, Wang F, Wang Y, Jin R. Atorvastatin improves endothelial function and cardiac performance in patients with dilated cardiomyopathy: the role of inflammation. Cardiovasc Drugs Ther. 2009;23(5):369-76.

8. Lieberman JA, Hammond FM, Barringer TA, Norton HJ, Goff DC, Jr., Bockenek WL, et al. Comparison of coronary artery calcification scores and National Cholesterol Education program guidelines for coronary heart disease risk assessment and treatment paradigms in individuals with chronic traumatic spinal cord injury. J Spinal Cord Med. 2011;34(2):233-40.

9. Stone NJ, Bilek S, Rosenbaum S. Recent National Cholesterol Education Program Adult Treatment Panel III update: adjustments and options. Am J Cardiol. 2005;96(4A):53E-9E.

10. Mohlenkamp S, Lehmann N, Schmermund A, Roggenbuck U, Moebus S, Dragano N, et al. Association of exercise capacity and the heart rate profile during exercise stress testing with subclinical coronary atherosclerosis: data from the Heinz Nixdorf Recall study. Clin Res Cardiol. 2009;98(10):665-76.

11. Zeman M, Zak A, Vecka M, Romaniv S. [Long-term hypolipidemic treatment of mixed hyperlipidemia with a combination of statins and fibrates]. Cas Lek Cesk. 2003;142(8):500-4.

12. Klosiewicz-Latoszek L, Szostak WB, Grzybowska B, BialobrzeskaPaluszkiewicz J, Wisniewska B, Stolarska I. Comparison of combined statin-fibrate treatment to monotherapy in patients with mixed hyperlipidemia. Kardiol Pol.2004;60(6):567-77.

13. Grundy SM, Vega GL, Tomassini JE, Tershakovec AM. Correlation of non-high-density lipoprotein cholesterol and low-density lipopro- tein cholesterol with apolipoprotein B during simvastatin + fenofibrate therapy in patients with combined hyperlipidemia (a subanalysis of the SAFARI trial).Am J Cardiol. 2009;104(4):548-53.

14. Brinton EA. Does the addition of fibrates to statin therapy have a favorable risk to benefit ratio? Curr Atheroscler Rep. 2008;10(1):25-32.

15. Mital S, Liao JK. Statins and the myocardium. Semin Vasc Med. 2004;4(4):377-84.

16. Hasegawa H, Yamamoto R, Takano H, Mizukami M, Asakawa M, Nagai T, et al. 3-Hydroxy-3-methylglutaryl coenzyme A reductase inhibitors prevent the development of cardiac hypertrophy and heart failure in rats. J Mol Cell Cardiol. 2003;35(8):953-60.

17. Nishikawa H, Miura S, Zhang B, Shimomura H, Arai H, Tsuchiya $\mathrm{Y}$, et al. Statins induce the regression of left ventricular mass in patients with angina. Circ J. 2004;68(2):121-5.

18. Sola S, Mir MQ, Lerakis S, Tandon N, Khan BV. Atorvastatin improves left ventricular systolic function and serum markers of inflammation in nonischemic heart failure. J Am Coll Cardiol. 2006;47(2):332-7.

19. Igarashi K, Tsuji M, Nishimura M, Horimoto M. Improvement of endothelium-dependent coronary vasodilation after a single LDL apheresis in patients with hypercholesterolemia. J Clin Apher. 2004;19(1):11-6.

20. Chen H, Ren JY, Xing Y, Zhang WL, Liu X, Wu P, et al. Short-term withdrawal of simvastatin induces endothelial dysfunction in patients with coronary artery disease: a dose-response effect dependent on endothelial nitric oxide synthase. Int J Cardiol. 2009;131(3):313-20.

21. Undas A, Celinska-Lowenhoff $M$, Lowenhoff T, Szczeklik A Statins, fenofibrate, and quinapril increase clot permeability and enhance fibrinolysis in patients with coronary artery disease. J Thromb Haemost. 2006;4(5):1029-36.

22. Schneider MP, Schmidt BM, John S, Schmieder RE. Effects of statin treatment on endothelial function, oxidative stress and inflammation in patients with arterial hypertension and normal cholesterol levels. J Hypertens. 2011;29(9):1757-64.

23. Blanco-Colio LM, Martin-Ventura JL, de Teresa E, Farsang C, Gaw A, Gensini G, et al. Increased soluble Fas plasma levels in subjects at high cardiovascular risk: Atorvastatin on Inflammatory Markers (AIM) study, a substudy of ACTFAST. Arterioscler Thromb Vasc Biol. 2007;27(1):168-74.

24. Polenova NV, Vaulin NA, Masenko VP, Iavelov IS, Gratsianskii NA. [Rosuvastatin and fenofibrate in patients with diabetes and low high density lipoprotein cholesterol: comparison of changes of lipid levels and some markers of inflammation]. Kardiologiia. 2009;49(2):9-14.

25. Bielecka-Dabrowa A, Mikhailidis DP, Hannam S, Aronow WS, Rysz J, Banach M. Statins and dilated cardiomyopathy: do we have enough data? Expert Opin Investig Drugs. 2011;20(3):315-23. 